S. Ullom

Nagoya Math. J.

Vol. 39 (1970), 141-148

\title{
INTEGRAL NORMAL BASES IN GALOIS EXTENSIONS OF LOGAL FIELDS
}

\author{
S. ULLOM
}

\section{Introduction}

Throughout this paper $F$ denotes a field complete with respect to a discrete valuation, $k_{F}$ the residue field of $F, K / F$ a finite Galois extension with Galois group $G=G(K / F)^{\dagger}$. The ring of integers $O_{K}$ of $K$ contains the (unique) prime ideal $\mathfrak{P}$; the collection of ideals $\mathfrak{P}^{n}$ for all integers $n$ are ambiguous ideals i.e. $G$-modules. E. Noether [3] showed $K / F$ tamely ramified implies $O_{K}$ has an $O_{F}$-normal basis, i.e. is isomorphic as an $O_{F} G$-module to $O_{F} G$ itself, $O_{F} G$ the group ring of $G$ over the ring $O_{F}$.

Define subgroups of $G$

$$
G_{i^{*}}=\left\{\sigma \in G \mid \forall \alpha \in O_{K}, \sigma \alpha-\alpha \in \mathfrak{P}^{i+1}\right\}, \quad i \geqslant 0
$$

and

$$
G_{i}^{*}=\left\{\sigma \in G \mid \forall \alpha \in K^{\times}, \sigma \alpha / \alpha \in 1+\mathfrak{P}^{i}\right\}, \quad i \geqslant 1 .
$$

Then $G_{i}{ } \supset G_{i+1}^{*} \supset G_{i+1}, i \geqslant 0$, with $G_{i+1}^{*}=G_{i+1^{*}}$ written $G_{i+1}$ if the residue field extension $k_{K} / k_{F}$ is separable [2, p. 35]. We show (Theorem 3) that an ambiguous ideal $\mathfrak{A}$ of $K$ has an $O_{F}$-normal basis iff the trace

$$
S_{K / K_{1}} \mathfrak{A}=\mathfrak{U} \cap K_{1},
$$

where $K_{1}$ is the fixed field of the subgroup $G_{1}^{*}$. This result is obtained from the Galois module structure of $\mathfrak{A} \otimes_{o_{F}} F$ (resp. $\mathfrak{A} \otimes_{o_{F}} k_{F}$ ) where $K / F$ is tamely ramified (resp. totally and wildly ramified).

Received November 11, 1968.

Revised June 6, 1969.

$\dagger$ Elements of Galois groups act on the left.

This research is supported by National Science Foundation Postdoctoral Fellowship Number 48037. 


\section{Tamely Ramified Extensions}

The following proposition generalizes a result given by Fröhlich [2, p. 22] for rings of integers.

Proposition 1. An ambiguous ideal $\mathfrak{A}$ of $K$ is $O_{F} G$-projective iff $\mathfrak{A}$ has an $O_{F}$-normal basis.

Proof. It suffices to consider $\mathfrak{A} O_{F} G$-projective. For any fractional ideal $\mathfrak{A}$ of $K$ we have $\mathfrak{A} F=K$. Further

$$
\mathfrak{A} F \cong \mathfrak{A} \otimes_{O_{F}} F,
$$

where $G$ acts on the righthand side of the above equation by

$$
\sigma(\alpha \otimes b)=(\sigma \alpha) \otimes b, \sigma \in G, \quad \alpha \in \mathfrak{A}, \quad b \in F .
$$

All isomorphisms are of $O_{F} G$-modules. By the normal basis theorem for fields

$$
K \cong F G \cong O_{F} G \otimes_{o_{F}} F .
$$

Since $O_{F}$ is a complete local domain, we may apply Swan's theorem [5, Corollary 6. 4, p. 567] to conclude

$$
\mathfrak{A} \cong O_{F} G .
$$

Definition. The extension $K / F$ is tamely ramified if the characteristic of $k_{F}$ does not divide $e\left(\mathfrak{p} O_{K}=\mathfrak{P}^{e}, \mathfrak{p}\right.$ the prime ideal of $\left.F\right)$ and the extension $k_{K} / k_{F}$ is separable. We say the extension is wildly ramified if it is not tamely ramified.

THEOREM 1. The extension $K / F$ is tamely ramified iff every ambiguous ideal of $K$ has an $O_{F}$-normal basis.

Proof. If $K / F$ is tamely ramified, then every ambiguous ideal of $K$ is $O_{F} G$-projective [6, Prop. 1. 3], and hence by Prop. 1 every ambiguous ideal of $K$ has an $O_{F}$-normal basis.

Conversely, if every ambiguous ideal of $K$ has an $O_{F}$-normal basis, then in particular $O_{K}$ has a normal basis; it follows that $S_{K / F} O_{K}=O_{F}$ and so $K / F$ is tamely ramified.

\section{Wildly Ramified Extensions}

The field $K$ has a normalized valuation 


$$
v: K^{\times} \rightarrow \boldsymbol{Z}
$$

with the property $v(\alpha+\beta) \geqslant \operatorname{Inf} v(\alpha), v(\beta)$ with equality if $v(\alpha) \neq v(\beta)$, and $v$ extends to $K$ by $v(0)=+\infty$. For an extension $K / F$ define the integers $f(K / F)=\left[k_{K}: k_{F}\right], e(K / F)=v\left(\pi_{F}\right), \pi_{F}$ a prime element of $F$; finally the different

$$
\mathfrak{D}(K / F)=\mathfrak{P}^{m(K / F)} \text {. }
$$

Proposition 2. Given the extension $K / F$ with $K_{1}$ the fixed field of $G_{1}^{*}$. If $f\left(K / K_{1}\right)>1$, then $m\left(K / K_{1}\right) \geqslant 2 e\left(K / K_{1}\right)-1$.

Proof. We use induction on $n,\left[K: K_{1}\right]=p^{n}$. Of course the characteristic of $k_{F}$ is $p$. Set $n=1$. Then

$$
\left[K: K_{1}\right]=f\left(K / K_{1}\right)=p, e\left(K / K_{1}\right)=1 .
$$

Since the non-trivial residue field extension is inseparable, $m\left(K / K_{1}\right) \geqslant 1$. Assume for all Galois extensions $K / F$ with $\left[K: K_{1}\right]=p^{n}$ and $f\left(K / K_{1}\right)>1$ that $m\left(K / K_{1}\right) \geqslant 2 e\left(K / K_{1}\right)-1$.

Consider $K / K_{1}$ Galois of order $p^{n+1}, n \geqslant 1, f\left(K / K_{1}\right)>1$. There exists a subfield $K^{\prime}, K \supset K^{\prime} \supset K_{1}$ with $\left[K: K^{\prime}\right]=p^{n}$ and $K^{\prime} / K_{1}$ Galois. By the tower formula for the different

$$
m\left(K / K_{1}\right)=m\left(K / K^{\prime}\right)+e\left(K / K^{\prime}\right) m\left(K^{\prime} / K_{1}\right) .
$$

If the subgroup $H \subset G$ fixes $K^{\prime}$, then [2, p. 35]

$$
H_{1}^{*}=H \cap G_{1}^{*}=H .
$$

Also

$$
m\left(K^{\prime} \mid K_{1}\right) \geqslant\left\{\begin{array}{cl}
2(p-1) & \text { if } e\left(K^{\prime} / K_{1}\right)=p \\
1 & \text { if } f\left(K^{\prime} / K_{1}\right)=p .
\end{array}\right.
$$

Suppose $f\left(K / K^{\prime}\right)>1$. Then by (2) we may apply the induction hypothesis to $K / K^{\prime}$. So by (1)

$$
\begin{aligned}
m\left(K / K_{1}\right) & \geqslant 2 e\left(K / K^{\prime}\right)-1+e\left(K / K^{\prime}\right) m\left(K^{\prime} / K_{1}\right) \\
& \geqslant 2 e\left(K / K_{1}\right)-1 \quad \text { by }(3) .
\end{aligned}
$$

If $f\left(K / K^{\prime}\right)=1$, then

$$
\left[K: K^{\prime}\right]=e\left(K / K^{\prime}\right)=e\left(K / K_{1}\right)
$$


and

$$
\left[K^{\prime}: K_{1}\right]=f\left(K^{\prime} / K_{1}\right)=f\left(K / K_{1}\right)
$$

Here $m\left(K / K^{\prime}\right) \geqslant 2\left(e\left(K / K^{\prime}\right)-1\right)$ and so we have the inequality

$$
m\left(K / K_{1}\right) \geqslant 3 e\left(K / K_{1}\right)-2 .
$$

Corollary. Given extension $K / F$. If for an ambiguous ideal $\mathfrak{A}=\mathfrak{P}^{s}$ of $K$ we have $S_{K / K_{1}} \mathfrak{A}=\mathfrak{A} \cap K_{1}$, then $f\left(K / K_{1}\right)=1, s \equiv 1 \bmod e\left(K / K_{1}\right)$ and $G_{2}=\{1\}$.

Proof. By [6]* we have for $m=m\left(K / K_{1}\right)$, etc.,

$$
[(m+s) / e]=1+[(s-1) / e]
$$

where $[x]$ denotes the greatest integer less than or equal to $x$. If $f>1$, then by Prop. 2

$$
[(2 e-1+s) / e] \leqslant 1+[(s-1) / e],
$$

which is impossible. Hence $f=1$, i.e., the residue field extension $k_{K} / k_{F}$ is separable. The remainder of the Corollary follows from [6, Theorem 2. 1].

$q . e . d$.

Cardinality of a finite set $S$ is Card $S$ and $R^{t}$ is the product of $t$ copies of a ring $R$. For a $G$-module $M, M^{G}$ denotes the group of fixed points under the action of $G$. When $f\left(K / K_{1}\right)=1, G_{i+1}^{*}=G_{i+1^{*}}, i \geqslant 0$, and we write $G_{i+1}$.

Proposition 3. Given the extension $K / K_{1}$ with $f\left(K / K_{1}\right)=1$ and $G_{2}=\{1\}$. Then the dimension of $(\mathfrak{P} / \mathfrak{p} \mathfrak{P})^{G_{1}}\left(\mathfrak{p}=\mathfrak{P} \cap K_{1}\right)$ as a vector space over $k=k_{K_{1}}$ is one.

Proof. The result is obviously true for $G_{1}=\{1\}$, so take $G_{1} \neq\{1\}$. Use the notation that for $\alpha, \beta \in O_{K}, \alpha \equiv \beta$ means $\alpha \equiv \beta \bmod \mathfrak{P}^{1+e}$, where $e=\operatorname{Card} G_{1}$; also characteristic of $k$ is $p$. Choose a prime element $\pi$ of $K$. Since $G_{2}=\{1\}$, for $\sigma \neq 1$

$$
\sigma \pi=\pi(1+\alpha(\sigma)), \quad \sigma \in G_{1}, \quad \alpha(\sigma) \in O_{K}, \quad v(\alpha(\sigma))=1 .
$$

For $1 \leq i \leq e-1, i=p^{c} n, p+n$, we have by (4) and the binomial expansion for $\sigma \neq 1$

* In [6] there is an a priori assumption of separability of residue field extensions; the results needed in this paper from [6] are seen immediately not to require this assumption. 


$$
\begin{aligned}
\sigma \pi^{i}-\pi^{i} & \equiv \pi^{i}\left(n \alpha(\sigma)^{p^{c}}+\cdots+\alpha(\sigma)^{i}\right) \\
& =\pi^{i+p^{c}}\left(n \beta(\sigma)^{p^{c}}\right)+\text { higher order terms }
\end{aligned}
$$

where $\alpha(\sigma)=\beta(\sigma) \pi$. Thus for $1 \leq i \leq e-1$

$$
v\left(\sigma \pi^{i}-\pi^{i}\right)=i+p^{c}
$$

and for $1 \leqslant i \leqslant e$

$$
\sigma \pi^{i}-\pi^{i} \equiv 0 \text { iff } i=e .
$$

Thus the dimension of $(\mathfrak{P} / \mathfrak{p} \mathfrak{P})^{G_{1}}$ is at least one. It remains to show given $\gamma \in O_{K}$ with $1 \leqslant v(\gamma)<e$, that there exists $\sigma \in G_{1}$ such that $\sigma \gamma \neq \gamma$. Since $\left[K: K_{1}\right]=e\left(K / K_{1}\right)$, the elements $\pi, \cdots, \pi^{e}$ are an $O_{K_{1}}$-basis of $\mathfrak{P}$ and hence their images in $\mathfrak{B} / \mathfrak{p} \mathfrak{B}$ are a $k$-basis. We may write $\gamma \equiv \sum_{i=1}^{e} a_{i} \pi^{i}, a_{i}=0$ or unit of $O_{K_{1}}$. For some $\sigma \neq 1$ set

$$
u=\operatorname{Inf}_{1 \leqslant i \leqslant e-1} v\left(a_{i}\left(\sigma \pi^{i}-\pi^{i}\right)\right) .
$$

Set

$$
\delta(\sigma)=\sum_{j=1}^{b} a_{\nu_{j}}\left(\sigma \pi^{\nu}-\pi^{\nu_{j}}\right), \nu_{1}<\cdots<\nu_{b} \text { if } b>1
$$

where the summation is over all $1 \leqslant i \leqslant e-1$ with $v\left(a_{i}\left(\sigma \pi^{i}-\pi^{i}\right)\right)=u$; set $\nu_{j}=p^{c_{j}} n_{j}, p+n_{j}$. Note $c_{1}>\cdots>c_{b}$ if $b>1$. From (5)

$$
\delta(\sigma)=\pi^{u} h(\beta(\sigma))+\text { higher order terms }(\delta(1)=\beta(1)=0)
$$

where the polynomial

$$
h(X)=\sum_{j=1}^{b} a_{\nu_{j}} n_{j} X^{p^{c_{j}}}
$$

Denote by $\bar{h}(X)$ the image of the polynomial $h(X)$ in the polynomial ring $k[X]$.

Assume $\forall \sigma \in G_{1}, \sigma \gamma \equiv \gamma$; then $\forall \sigma \in G_{1}, v(\delta(\sigma)) \geqslant u+1$ since $u \leqslant e$. Hence $\forall \sigma \in G_{1}, v(h(\beta(\sigma))) \geqslant 1$. In general we have the homomorphism of $G_{1}$ into the additive group of $O_{K} / \mathfrak{P}$ given by $\sigma \rightarrow \bar{\beta}(\sigma)$ when $\sigma \in G_{1}$ and $\bar{\beta}(\sigma)$ is the image of $\beta(\sigma) \in O_{K}$ in $O_{K} / \Re$. The kernel is $G_{2}$ which is trivial by hypothesis. For another prime element $\pi$ of $K$, the $\bar{\beta}(\sigma)$ are determined up to multiplication by a unit of $O_{K} / \mathfrak{F}$, but we are interested only in the number of distinct $\bar{\beta}(\sigma), \sigma \in G_{1}$. The condition $\forall \sigma \in G_{1}, v(h(\beta(\sigma))) \geqslant 1$, becomes in the field $O_{K} / \mathfrak{S}$

$$
\bar{h}(\bar{\beta}(\sigma))=0 \quad \forall \sigma \in G_{1} .
$$


For any choice of prime element $\pi$ of $K$ the polynomial $\bar{h}(X)$ has degree less than or equal to $e / p$ but has $e$ (distinct) roots by (6). This is impossible since $\vec{h}(X)$ is not the zero element of $k[X]$; so there exists $\sigma \in G_{1}$ such that $\sigma \gamma \neq \gamma$.

For completeness we include a proof of the following well-known proposition; see e.g. [1, §3, Exercise 13] for a partial statement.

Proposition 4. Let $R$ be a discrete valuation ring with residue field $k=R / \mathfrak{p}$ of characteristic $p>0$. Let $G$ be a finite $p$-group and $M$ an $R G$-module which is $R$-projective and of $R$-rank Card $G$. The following are equivalent:

(i) $\operatorname{dim}(M / \mathfrak{p} M)^{G}=1(\operatorname{dim}=$ vector space dimension over $k)$.

(ii) $M \cong R G$ as $R G$-modules.

Proof. (ii) implies (i) is clear, so we consider only (i) implies (ii). Let $W$ be a $k G$-module with $\operatorname{dim} W$ finite, $I$ the two-sided nilpotent ideal which is the kernel of the augmentation homomorphism

$$
\varepsilon: k G \rightarrow k, \varepsilon\left(\sum a_{o} \sigma\right)=\sum a_{\sigma}, a_{\sigma} \in k \text {. }
$$

From now on we will assume $\operatorname{dim} W / I W$ to be one. Define the map $\phi$ as the composite

$$
k \underset{k \rightarrow k}{\stackrel{\approx}{\rightarrow} W} W \mid I W
$$

We have the diagram of $k G$-modules with exact row

$$
O \longrightarrow I W \longrightarrow W \stackrel{\psi}{\longrightarrow} \underset{\downarrow}{\stackrel{k}{\downarrow} \phi}(I W \longrightarrow O .
$$

There exists a $k G$-linear map $\theta: k G \rightarrow W$ with $\psi \theta=\phi$ since $k G$ is projective over itself. Use $I$ nilpotent to show $\theta$ surjective. Further if $\operatorname{dim} k G=\operatorname{dim} W$, $\theta$ is also injective and therefore an isomorphism.

Thus if we set $M / \mathfrak{p} \quad M=W$, we have $M / \mathfrak{p} M \cong k G$. Use $M$ is $R$-projective and the standard argument with Nakayama's lemma to show $M \cong R G$.

$q . e . d$.

Putting together Propositions 3 and 4 and noting $\mathfrak{F}$ is $O_{K_{1}}$-projective, we have proved the following theorem. 
Theorem 2. Given the extension $K / K_{1}$ with $f\left(K / K_{1}\right)=1$ and $G_{2}=\{1\}$. Then the ambiguous ideal $\mathfrak{P}$ of $K$ has an $O_{K_{1}}$-normal basis.

\section{Arbitrary Extensions}

Given a commutative ring $R$ with 1 and finite group $G$. An $R G$-module $M$ is relatively $R G$-projective* if there exists an $R$-endomorphism $\phi$ of $M$ with $S_{G}(\phi)=1_{M}$, i.e.

$$
\sum_{\sigma \in G} \sigma\left(\phi\left(\sigma^{-1} m\right)\right)=m \quad \forall m \in M
$$

Proposition 5. Given an extension $K / F, H$ a subgroup of the Galois group $G$ with fixed field $L$. Suppose $L / F$ is tamely ramified. If an ambiguous ideal $\mathfrak{A}$ of $K$ is relatively $O_{L} H$-projective, then it is $O_{F} G$-projective.

Proof. By hypothesis there exists an $O_{L}$-endomorphism $\psi$ of $\mathfrak{A}$ with $S_{H}(\psi)=1_{\mathscr{U}} . \quad L / F \quad$ tamely ramified implies there exists $\beta \in O_{L}$ with $S_{L / F}(\beta)=1$. Denote also by $\beta$ the endomorphism of $\mathfrak{A}$ given by multiplication by $\beta$. Then for the $O_{F}$-endomorphism $\psi \cdot \beta$ of $\mathfrak{A}$ a short computation shows $S_{G}(\psi \cdot \beta)=1 \mathfrak{a}$. So $\mathfrak{A}$ is relatively $O_{F} G$-projective. On the other hand, $\mathfrak{A}$ is $O_{F}$-projective and thus $O_{F} G$-projective [4, Prop. 2. 3, p. 702].

We can now prove the main result.

Theorem 3. An ambiguous ideal $\mathfrak{A}$ of the extension $K$ over $F$ has an $O_{F^{-}}$ normal basis iff $S_{K / K_{1}} \mathfrak{A}=\mathfrak{A} \cap K_{1}$.

Proof. If $\mathfrak{A}$ has a normal basis, then it is easy to see that $S_{K / K_{1}} \mathfrak{A}=\mathfrak{X} \cap K_{1}$. Conversely, assume $S_{K / K_{1}} \mathfrak{A}=\mathfrak{A} \cap K_{1}$. Take $G_{1}^{*} \neq\{1\}$, otherwise we are done by Theorem 1. By the Corollary to Prop. 2, $f\left(K / K_{1}\right)=1, \mathfrak{A}=\mathfrak{P}^{s} \cong \mathfrak{P}$ as $O_{K_{1}} G_{1}$-modules. By Theorem $2 \mathfrak{P} \cong O_{K_{1}} G_{1}$. Since $K_{1} / F$ is tamely ramified, we apply Prop. 5 to conclude $\mathfrak{A}$ is $O_{F} G$-projective. Then Prop. 1 shows $\mathfrak{A} \cong O_{F} G$.

\section{ReFERENCES}

[ 1 ] N. Bourbaki, Algèbre Commutative, Actualités scientifiques et industrielles 1290, Hermann, Paris, 1961, Chapitre 2.

[2] A. Fröhlich, Local Fields, Algebraic Number Theory, Academic Press, London, 1967. rings.

* See [4] for the needed results on relatively (weakly) projective modules over group 
[ 3 ] E. Noether, Normalbasis bei Körpern ohne höhere Verzweigung, J. reine angew. Math. 167 (1931), 147-152.

[ 4 ] D.S. Rim, Modules over finite groups, Ann. of Math. 69 (1959), 700-712.

[ 5 ] R. Swan, Induced representations and projective modules, Ann. of Math. 71 (1960), 552-578.

[6] S. Ullom, Normal bases in Galois extensions of number fields, Nagoya Math. J. 34 (1969), 153-167.

University of Illinois

Urbana, Illinois 